\title{
Small Forbidden Configurations II
}

\author{
Richard Anstee* \\ Ron Ferguson ${ }^{\dagger}$ \\ Mathematics Department \\ The University of British Columbia \\ Vancouver, B.C. Canada V6T 1Z2 \\ anstee@math.ubc.ca \\ Attila Sali $\ddagger$ \\ Department of Computer Science \\ Indiana University-Purdue University Fort Wayne \\ Fort Wayne, Indiana, 46805-1499
}

AMS Subject Classification: 05D05 (primary), 05C20, 05C90 (secondary)

Submitted: July 20, 2000; Accepted: October 3, 2000

\begin{abstract}
The present paper continues the work begun by Anstee, Griggs and Sali on small forbidden configurations. In the notation of $(0,1)$-matrices, we consider a (0,1)-matrix $F$ (the forbidden configuration), an $m \times n(0,1)$-matrix $A$ with no repeated columns which has no submatrix which is a row and column permutation of $F$, and seek bounds on $n$ in terms of $m$ and $F$. We give new exact bounds for some $2 \times l$ forbidden configurations and some asymptotically exact bounds for some other $2 \times l$ forbidden configurations. We frequently employ graph theory and in one case develop a new vertex ordering for directed graphs that generalizes Rédei's Theorem for Tournaments. One can now imagine that exact bounds could be available for all $2 \times l$ forbidden configurations. Some progress is reported for $3 \times l$ forbidden configurations. These bounds are improvements of the general bounds obtained by Sauer, Perles and Shelah, Vapnik and Chervonenkis.
\end{abstract}

\footnotetext{
${ }^{*}$ Research supported in part by NSERC

${ }^{\dagger}$ Research supported in part by NSERC

$\ddagger$ This research was done while the third author visited the University of British Columbia supported by the first author's NSERC grant. Permanent address: Alfréd Rényi Institute of Mathematics, The Hungarian Academy of Sciences, Budapest, P.O.B. 127 H-1364, Hungary, sali@renyi .hu.
} 


\section{Introduction}

This paper continues investigations of Anstee, Griggs, Sali [4] into extremal set problems arising from forbidding a single configuration. The reader might consider the analogy with the celebrated results of Erdös and Stone [5] who determined asymptotic bounds on the number of edges (in terms of the number of vertices) in a graph avoiding a single given subgraph (based on the chromatic number of the forbidden subgraph). Our results provide bounds that are remarkably accurate for small forbidden configurations but we still have some small forbidden configurations for which we do not know the asymptotic bounds. The results are examples of a general pattern as yet not fully understood to the same extent as the Erdös-Stone results.

A natural notation for these problems is $(0,1)$-matrices. Forbidden configurations have been studied by various authors for a long time, because a great number of combinatorial objects can be encoded as $(0,1)$-matrices with forbidden substructures. We will use the term configuration (the combinatorial equivalent of submatrix) as follows. For a matrix $F$, we say a matrix $A$ has no configuration $F$ if $A$ has no submatrix which is a row and column permutation of $F$. Let $F$ be a $k \times l(0,1)$-matrix and let $A$ be an $m \times n(0,1)$ matrix with no repeated columns (such matrix is called simple). The maximum number of columns of a simple matrix $A$ of $m$ rows with no configuration $F$ will be denoted by forb $(m, F)$. Obviously forb $(m, F) \leq 2^{m}$ but more can be done.

This paper is noteworthy in establishing a number of best possible bounds and some asymptotically best possible bounds for various $2 \times l$ forbidden configurations $F$. Careful counting arguments are used and then reused in searching for examples close to the bounds. We are getting close to providing exact bounds for any $2 \times l$ configuration $F$, which before seemed hopeless. Section 2 focuses on $2 \times l F$ yielding linear bounds. Section 3 focuses on $2 \times l F$ yielding quadratic bounds. The final section provides a new result for general $F$, (multiple copies of the identity matrix) which in particular shows that for

$$
F=\left[\begin{array}{llllll}
1 & 1 & 0 & 0 & 0 & 0 \\
0 & 0 & 1 & 1 & 0 & 0 \\
0 & 0 & 0 & 0 & 1 & 1
\end{array}\right]
$$

that $\operatorname{forb}(m, F)=O\left(m^{7 / 3}\right)$. We would tend to believe that forb $(m, F)=\Theta\left(m^{2}\right)$, and must leave this as an open problem.

We extensively use graph theory for $2 \times l$ forbidden configurations $F$. Lemma 2.1, which generalizes Rédei's result for hamiltonian paths in tournaments, provides an interesting new vertex ordering for directed graphs. The applications suggest that maybe there is a hypergraph generalization to aid in obtaining bounds for general forbidden configurations.

The following result of Füredi is a general asymptotic bound.

Theorem $1.1([9])$ Let $F$ be a $k \times l(0,1)$-matrix. Then

$$
\operatorname{forb}(m, F)=O\left(m^{k}\right) .
$$


This is best possible for $F$ being the $k \times 2$ matrix of ones. To obtain a proof one can use the following fundamental result of Sauer, Perles and Shelah, Vapnik and Chervonenkis. Let $K_{k}$ denote the $k \times 2^{k}$ submatrix of all possible columns of size $k$.

Theorem $1.2([10,12,13])$

$$
\operatorname{forb}\left(m, K_{k}\right)=\left(\begin{array}{c}
m \\
k-1
\end{array}\right)+\left(\begin{array}{c}
m \\
k-2
\end{array}\right)+\ldots\left(\begin{array}{c}
m \\
0
\end{array}\right) .
$$

It is easy to see that the bound of Theorem 1.2 is sharp: take $A$ to be the matrix of all columns with $k-1$ or fewer 1's. To obtain the asymptotic estimate for arbitrary forbidden configurations $F$ one can do the following. Given a matrix $A$ with at least

$$
\left(2^{k}\right)(l-1)\left(\begin{array}{c}
m \\
k
\end{array}\right)+\left(\begin{array}{c}
m \\
k-1
\end{array}\right)+\left(\begin{array}{c}
m \\
k-2
\end{array}\right)+\ldots\left(\begin{array}{c}
m \\
0
\end{array}\right)+1
$$

columns, we take any $\left(\begin{array}{c}m \\ k-1\end{array}\right)+\left(\begin{array}{c}m \\ k-2\end{array}\right)+\ldots\left(\begin{array}{c}m \\ 0\end{array}\right)+1=$ forb $\left(m, K_{k}\right)+1$ columns which then contain the configuration $K_{k}$ in $2^{k}$ columns. Permute the columns so that these $2^{k}$ columns are the first ones. Repeat on the remaining columns until one has $(l-1)\left(\begin{array}{c}m \\ k\end{array}\right)+1$ such $K_{k}$ 's and hence by the Pidgeonhole Principle, at least $l$ in the same $k$ subset of rows and hence a copy of $F$.

One can obtain best possible or at least more accurate bounds (see [3]). For example, we have for $F$ being the $k \times 2$ matrix of ones

$$
\operatorname{forb}(m, F)=\left(\begin{array}{c}
m \\
k
\end{array}\right)+\left(\begin{array}{c}
m \\
k-1
\end{array}\right)+\ldots\left(\begin{array}{c}
m \\
0
\end{array}\right)=\Theta\left(m^{k}\right) .
$$

Also if $F$ has no repeated columns, then

$$
\operatorname{forb}(m, F) \leq\left(\begin{array}{c}
m \\
k-1
\end{array}\right)+\left(\begin{array}{c}
m \\
k-2
\end{array}\right)+\ldots\left(\begin{array}{c}
m \\
0
\end{array}\right)=\Theta\left(m^{k-1}\right)
$$

but the bound need not be best-possible. For other results or generalizations see $[1,2,6,7]$.

\section{Linear Bounds}

Careful structural analysis has resulted in bounds that are new and surprisingly exact. The following graph theory argument is the key to the exact bound in the Theorem 2.2 that follows. One can envision this as a generalization of Rédei's result [11] that a tournament has a hamiltonian path. While this result is for directed graphs, it is interesting to contemplate whether there are $k$-uniform hypergraph analogues useful for $k \times l F$ and in particular to use in Theorem 4.1. 
Lemma 2.1 Let $D=(N, A)$ be a directed graph. There is an ordering of the vertices $N$ as $1,2, \ldots m$ where $m=|N|$ and a subset $T \subseteq A$ consisting of a collection of vertex disjoint indirected trees $T$ with the following property. Let $D_{i}$ denote the subgraph of $D$ induced by the vertices $\{i, i+1, \ldots m\}$. For each pair $i, j, 1 \leq i<j \leq m$ either there is a directed path in $D_{i}$ from $i$ to $j$ or there is a $k$ with $i \leq k \leq m$ so that there is a directed path from $i$ to $k$ in $D_{i}$ and there is no edge in $D$ from $k$ to $j$.

Proof: We proceed by constructing a forest of indirected trees $T$ from $D$ and a vertex ordering in the following way. As vertices are deleted, they enter the vertex ordering; namely the $k$ th vertex deleted is labelled $k$. We start with $T=\emptyset$. At the $j$ th stage we form a directed path $P_{j}$ from $P_{j-1}$ by extending it. Let $P_{j}=i_{j, 1} \rightarrow i_{j, 2} \rightarrow \ldots \rightarrow i_{j, l_{j}}$. It will have what we call the two-maximal property that for a vertex $k \notin P_{j}$ (and not yet deleted) there is no edge $k \rightarrow i_{j, 1}$, there is no pair of edges $i_{j, t} \rightarrow k, k \rightarrow i_{j, t+1}$ for all $t$ with $1 \leq t \leq l_{j}-1$ and there is no edge $i_{j, l_{j}} \rightarrow k$. We initially start with $P_{0}=\emptyset$.

At the $n+1$ st step we look at $P_{n}$. In the case $P_{n}=\emptyset$, we simply choose $P_{n+1}$ as a two-maximal path in the remaining graph ( $P_{n+1}$ could consist of a single vertex). For $P_{n}=i_{n, 1} \rightarrow i_{n, 2} \rightarrow \ldots \rightarrow i_{n, l_{n}}$, we find the smallest index $k_{n}$ such that there is an edge $j \rightarrow i_{n, k_{n}}$ for a vertex $j$ (not in $P_{n}$ and not already deleted). If there is no vertex $k_{n}$, then set $P_{n+1}=\emptyset$ while deleting the vertices $i_{n, 1}, i_{n, 2}, \ldots i_{n, l_{n}}$ in turn. Otherwise add the edges $i_{n, 1} \rightarrow i_{n, 2}, i_{n, 2} \rightarrow i_{n, 3}, \ldots i_{n, k_{n}-1} \rightarrow i_{n, k_{n}}$ to $T$ and delete the vertices $i_{n, 1}, i_{n, 2}, \ldots i_{n, k_{n}}$ in turn (note that by the two maximal property for $P_{n}$ that $k_{n}>1$ ).

Extend $P_{n}$ to a two-maximal path $i_{n+1,1} \rightarrow \ldots \rightarrow i_{n+1, e_{n+1}} \rightarrow i_{n, k_{n}} \rightarrow \ldots \rightarrow i_{n, l_{n}}$. This we relabel as our new path $P_{n+1} i_{n+1,1} \rightarrow i_{n+1,2} \rightarrow \ldots \rightarrow i_{n+1, l_{n+1}}$.

This process continues until there are no vertices left. Does the resulting ordering and set $T$ have the desired property? Note that each path $P_{n}$ has all its edges eventually enter $T$ and the vertex ordering respects the vertex ordering in the path. Choose any $s$ satisfying $1 \leq s \leq k_{n}-1$. Using the two-maximal property, we verify that for every vertex $j$ not in $P_{n}$ and yet still undeleted we note that either there is a vertex $i_{n, t}$ in $P_{n}$ (with $s \leq t \leq l_{n}$ ) where there is no edge (of $D$ ) joining $j, i_{n, t}$ or we get a contradiction. This follows since by the choice of $k_{n}$, we have $i_{n, s} \rightarrow j$ and using two-maximality we get that $i_{n, s+1} \rightarrow j, i_{n, s+2} \rightarrow j, \ldots, i_{n, l_{n}} \rightarrow j$ and the final arc contradicts the two-maximality of $P_{n}$.

We obtain a new exact result.

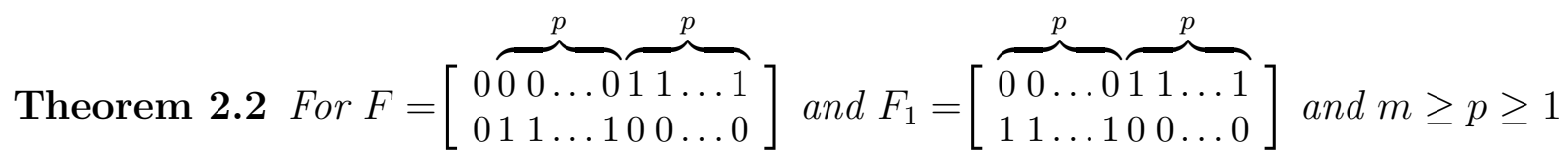

$$
\operatorname{forb}(m, F)=\operatorname{forb}\left(m, F_{1}\right)=(m-1) p+2 .
$$

Proof: We first show that forb $(m, F) \leq(m-1) p+2$. Let $A$ be a simple matrix of $m$ rows not containing $F$. We construct a directed graph $D$ using row numbers of $A$ as vertices and adding the edges $i \rightarrow j$ for each $i$ and $j$ such that the number of $\left[\begin{array}{l}0 \\ 1\end{array}\right]$ connections between 
row $i$ and row $j$ is less than $p$. As a result of forbidding $F$, we see that if $i, j$ are not joined in $D$ then row $i$ over row $j$ lacks the column $\left[\begin{array}{l}0 \\ 0\end{array}\right]$.

We use Lemma 2.1 to obtain a vertex ordering for $D$ and a forest of indirected trees $T$ consisting of at most $m-1$ edges. Rearrange the rows of $A$ in accordance with the ordering. The number of columns of $A$ with an entry $\left[\begin{array}{l}0 \\ 1\end{array}\right]$ in row $i$ over row $j$ for some edge $i \rightarrow j$ in our forest $T$ is at most $(m-1)(p-1)$, using the definition of $i \rightarrow j$.

There are at most $m+1$ columns which for each edge $i \rightarrow j$ of $T$, do not have an entry $\left[\begin{array}{l}0 \\ 1\end{array}\right]$ in row $i$ over row $j$. To see this note that apart from the column of 1 's, each such column must have a highest row $i$ with a 0 , namely 1 's in rows $1,2, \ldots, i-1$ and a 0 in row $i$. Applying the lemma we consider any row $j$ with $i<j \leq m$. If there is a directed path from $i$ to $j$ then all the entries in the rows corresponding to the vertices of the path are forced to be 0's. If there is a directed path from $i$ to $k$ and there is no edge in $D$ from $k$ to $j$ then the entry in row $k$ is forced to be 0 and then the entry in row $j$ is forced to be 1 (by the lack of an edge from $j$ to $k$ in $D$ ). Thus there are at most $(m-1)(p-1)+(m+1)=(m-1) p+2$ columns in $A$.

We complete our proof by showing forb $\left(m, F_{1}\right) \geq(m-1) p+2$ using a construction. We form $(m-1)(p-1)$ columns $C_{i j}$ for $1 \leq i \leq p-1$ and $1 \leq j<i$ and $i+1<j \leq p$ with $C_{i j}$ having 1's in rows $1,2, \ldots, i-1,0$ in row $i, 1$ in row $i+1$, and 0 's in rows $i+1, i+3, \ldots, m$. We then add the $m+1$ columns containing no submatrix $\left[\begin{array}{l}0 \\ 1\end{array}\right]$ to obtain a simple $m \times((m-1) p+2)$ matrix with no configuration $F_{1}$.

Some of the proof for the bounds for forbidden configurations proceed by what we call the standard argument, made in reference to a particular row of a simple $m \times n$ matrix. We first bring this row to the top of the matrix and then rearrange the columns to produce a matrix in the following form:

$$
\left[\begin{array}{cccc}
11 \ldots 1 & 11 \ldots 1 & 00 \ldots 0 & 00 \ldots 0 \\
B_{1} & B_{2} & B_{2} & B_{3}
\end{array}\right]
$$

where $B_{1}, B_{2}$ and $B_{3}$ are each matrices with $m-1$ rows, but $B_{1}$ and $B_{3}$ have no identical columns. The matrix

$$
\left[B_{1} B_{2} B_{3}\right]
$$

is a simple matrix. This process may be extended for multiple rows to obtain new results and simplified proofs of old results as in the following result for which a weaker linear bound (of $(2 p-2) m$ ) is given as Theorem 2.2 in [4].

Theorem 2.3 For $F=\left[\begin{array}{lllllll}0 & \overbrace{1}^{1} & \ldots & 1 & 0 & 1 \\ 0 & 0 & 0 & \ldots & 0 & 1 & 1\end{array}\right]$, forb $(m, F) \leq\left(p-\frac{1}{2}\right) m+1$.

Proof: Let $A$ be an $m \times n$ simple matrix with no configuration $F$. If a pair of rows has the configuration $K_{2}$, then that pair of rows has no configuration $F^{\prime}=\left[\begin{array}{cccc}0 & \overbrace{0}^{0} & \ldots & 0 \\ 1 & 1 & \ldots & 1\end{array}\right]$. 
Let $C$ be a maximal set of rows so that for every pair $i, j \in C$, the rows $i, j$ do not have the configuration $F^{\prime}$. We may assume $|C|>1$ since if there are no pair of rows avoiding $F^{\prime}$ then $A$ avoids $K_{2}$ and so $n \leq m+1$ by Theorem 1.2 .

We may reorder the rows of $A$ so that the $k$ rows of $C$ are first and then decompose $A$ as

$$
A=\left[\begin{array}{ccc}
0^{\prime} s & B & 1^{\prime} s \\
D & E & G
\end{array}\right]
$$

where every column in the $k \times l$ matrix $B$ contains the configuration $\left[\begin{array}{l}0 \\ 1\end{array}\right]$ and so in fact contains at least $k-1\left[\begin{array}{l}0 \\ 1\end{array}\right]$ 's. Now $B$ has no configuration $F^{\prime}$ and so we deduce $(k-1) l \leq$ $2(p-1)\left(\begin{array}{l}k \\ 2\end{array}\right)$ since there are at most $2(p-1)$ configurations $\left[\begin{array}{l}0 \\ 1\end{array}\right]$. This yields $l \leq(p-1) k$.

We find that at most one column can be in common to both $D$ and $G$ since if there were two columns $\alpha, \gamma$ in both $D$ and $G$ then in some row $t$ there is the configuration $\left[\begin{array}{ll}0 & 1\end{array}\right]$ in both $D$ and $G$. But then for every $i \in C$, there is a $K_{2}$ on rows $i, t$ and hence no $F^{\prime}$ on rows $i, t$, which contradicts that $C$ is a maximal set.

Thus we can delete from $A$ the $k$ rows of $C$ and at most $(p-1) k+1$ columns to obtain a simple matrix $A^{\prime}$. Thus

$$
\operatorname{forb}(m, F) \leq \operatorname{forb}(m-k, F)+(p-1) k+1 .
$$

For $k \geq 2$ we have $\frac{(p-1) k+1}{k} \leq p-\frac{1}{2}$ and so we obtain the bound.

The following constructions provide lower bounds on forb $(m, F)$ for $F$ as above.

Proposition 2.4 For $F=\left[\begin{array}{lllll}0 & \overbrace{1}^{1} & 1 & \ldots & 1 \\ 0 & 0 & 0 & \ldots & 0\end{array}\right]$ or $\left[\begin{array}{lllll}0 & \overbrace{1}^{1} & 1 & \ldots & 1 \\ 1 & 0 & 0 & \ldots & 0\end{array}\right]$ and $p$ odd and $p \geq 9$

$$
\text { forb }(m, F) \geq\left(\frac{p}{2}+\frac{3}{2}+\frac{2}{p+1}\right) m+\mathrm{O}(1),
$$

while for $p$ even and $p \geq 12$

$$
f o r b(m, F) \geq\left(\frac{p}{2}+\frac{4}{3}+\frac{2(1-(p \quad(\bmod 6))}{p}\right) m+\mathrm{O}(1) .
$$

Proof: For each $p$, the construction below determines a number $l$ and a simple $l$-rowed matrix $K$. Inductively we expand a simple $m$-rowed matrix $A_{m}$ avoiding $F$ to an $m+l$ rowed matrix $A_{m+l}$ avoiding $F$ as follows:

$$
A_{m+l}=\left[\begin{array}{cc}
A_{m} & 1 ' s \\
1 ' s & K
\end{array}\right]
$$

The new matrix has $\left|A_{m}\right|+|K|$ columns.

The matrix $K_{l}=\left[K_{l}^{0}, K_{l}^{1}, K_{l}^{2}, K_{l}^{l-2}, K_{l}^{l-1}\right]$ contains $l^{2}+l+1$ columns and $2 l-2\left[\begin{array}{l}0 \\ 1\end{array}\right]$ 's 
and $2 l-2\left[\begin{array}{l}1 \\ 0\end{array}\right]$ 's in each pair of rows.

For $p$ odd, we choose $l=(p+1) / 2$ and $K=K_{l}$. This matrix contains $\left(p^{2}+4 p+7\right) / 4$ columns and has exactly $p-1\left[\begin{array}{l}0 \\ 1\end{array}\right]$ 's and $\left[\begin{array}{l}1 \\ 0\end{array}\right]$ 's in each pair of rows. This gives a growth rate of $\left(\frac{p^{2}+4 p+7}{4}\right) /\left(\frac{p+1}{2}\right)=\left(\frac{p}{2}+\frac{3}{2}+\frac{2}{p+1}\right)$ per added row.

When $p$ is even, we choose $l=p / 2$. $K_{l}$ now has $p-2\left[\begin{array}{l}0 \\ 1\end{array}\right]$ 's and $\left[\begin{array}{l}1 \\ 0\end{array}\right]$ 's in each pair of rows. We produce $K$ by augmenting $K_{l}$ with $\left\lfloor\frac{p}{6}\right\rfloor$ columns from $K_{p / 2}^{3}$ so that we add no more that an extra 1 to each row of the $p / 2$ rows; thus, we still have fewer than $p-1\left[\begin{array}{l}0 \\ 1\end{array}\right]$ 's and $\left[\begin{array}{l}1 \\ 0\end{array}\right]$ 's in each pair of rows of $K$.

$K$ has $\frac{p^{2}}{4}+\frac{2 p}{3}+1-(p(\bmod 6))$ columns giving a growth rate of $\frac{p}{2}+\frac{4}{3}+\frac{2(1-(p(\bmod 6)))}{p}$ columns per added row.

The following result includes Theorem 2.5 of [4].

Corollary 2.5 For any submatrix $F_{s}$ of $F=\left[\begin{array}{lllll}1 & 1 & 1 & 0 & 0 \\ 1 & 0 & 0 & 1 & 0\end{array}\right]$ containing either the configuration $\left[\begin{array}{lll}1 & 1 & 0 \\ 0 & 0 & 1\end{array}\right]$ or $\left[\begin{array}{lll}0 & 0 & 0\end{array}\right]$ or $\left[\begin{array}{lll}1 & 1 & 1\end{array}\right]$,

$$
\operatorname{forb}\left(m, F_{s}\right)=\left\lfloor\frac{3}{2} m\right\rfloor+1
$$

Proof: The bound forb $(m, F) \leq\left\lfloor\frac{3}{2} m\right\rfloor+1$ follows from Theorem 2.3. Since forb $\left(m, F_{s}\right) \leq$ forb $(m, F)$, we have only to prove forb $\left(m, F_{s}\right) \geq\left\lfloor\frac{3}{2} m\right\rfloor+1$. Note that the matrices $A_{m}$ constructed in Proposition 2.4 do not contain the configuration $\left[\begin{array}{lll}1 & 1 & 0 \\ 0 & 0 & 1\end{array}\right]$. This gives the first case. For the second case, we construct the $m \times\left(\left\lfloor\frac{3}{2} m\right\rfloor+1\right)$ matrices $C_{m}$ inductively starting with $C_{1}=\left[\begin{array}{ll}0 & 1\end{array}\right]$ and $C_{2}=\left[\begin{array}{llll}0 & 1 & 0 & 1 \\ 0 & 0 & 1 & 1\end{array}\right]$ as before. $C_{m}$ is constructed by adding a row of 1's under the matrix $C_{m-1}$ and the $m \times 1$ column $\left[\begin{array}{c}1 \\ 1 \\ \vdots \\ 1 \\ 1 \\ 0\end{array}\right]$ for $m$ odd, the $m \times 2$ matrix $\left[\begin{array}{cc}1 & 1 \\ 1 & 1 \\ \vdots & \vdots \\ 1 & 1 \\ 1 & 0 \\ 0 & 0\end{array}\right]$ for $m$ even. These matrices do not contain the configuration $\left[\begin{array}{lll}0 & 0 & 0\end{array}\right]$. The complementary construction works for $\left[\begin{array}{lll}1 & 1 & 1\end{array}\right]$. The following theorem gives a new exact bound using a similar method. 
Theorem 2.6 For $F=\left[\begin{array}{llllll}0 & 1 & 1 & 1 & 0 & 1 \\ 0 & 0 & 0 & 0 & 1 & 1\end{array}\right]$ and $m \geq 3$

$$
\operatorname{forb}(m, F)=\left\lfloor\frac{7}{3} m\right\rfloor+1
$$

Proof: We construct the matrices $A_{m}$ inductively as follows. $A_{3}=K_{3} . A_{4}$ and $A_{5}$ have the constant columns along with those with only one 0 or 1 . For $m \geq 6$ we obtain $A_{m}$ from $A_{m-3}$ by adding 7 columns in the following way:

$$
A_{m}=\left[\begin{array}{cccccccc}
A_{m-3} & \multicolumn{8}{c}{1 ' \mathrm{~s}} & & \\
& 0 & 0 & 0 & 1 & 1 & 1 & 1 \\
0 ' \mathrm{~s} & 0 & 1 & 1 & 0 & 0 & 1 & 1 \\
& 1 & 0 & 1 & 0 & 1 & 0 & 1
\end{array}\right] .
$$

None of these simple matrices contains the configuration $F$, and each has $\left\lfloor\frac{7}{3} m\right\rfloor+1$ columns, establishing the quantity on the right as a lower bound.

We now show that the upper bound using the proof ideas of Theorem 2.3. We use induction on $m$. If we can find a $C$ as in Theorem 2.3 with $|C|=k \geq 3$ then $\frac{(p-1) k+1}{k} \leq \frac{7}{3}$ (using $p=3$ ). Hence we would have forb $(m, F) \leq \operatorname{forb}(m-k, F)+\frac{7}{3} k$ and so we obtain our bound. Hence we may assume that the largest $C$ that can be found is of size 2 and that the $2 \times l$ matrix $B$ in (1) has $l=4$ since for $l \leq 3$ we could delete 2 rows and at most 4 columns yielding the desired bound by induction. This forces $B$ to be the matrix $\left[\begin{array}{l}0011 \\ 1100\end{array}\right]$ apart from column order. We may rearrange $A$ to obtain

$$
\mathrm{A}=\left[\begin{array}{ccc|ccc|cccc|ccc|ccc}
0 & \ldots & 0 & 0 & \ldots & 0 & 1 & 1 & 0 & 0 & 1 & \ldots & 1 & 1 & \ldots & 1 \\
0 & \ldots & 0 & 0 & \ldots & 0 & 0 & 0 & 1 & 1 & 1 & \ldots & 1 & 1 & \ldots & 1 \\
& B_{1} & & & B_{2} & & E & & & B_{2} & & & B_{3} &
\end{array}\right]
$$

where $\left[B_{1} B_{2}\right]=D$ and $\left[B_{2} B_{3}\right]=G$ and $B_{2}$ are the columns in common. We find that $B_{2}$ has exactly one column. Our argument in Theorem 2.3 showed that there is at most one column and if there is no column then we could delete the first two rows and the 4 columns (containing E) and obtain a simple matrix so forb $(m, F) \leq \operatorname{forb}(m-2, F)+4$ which yields the bound.

We find a row such that the first 2 entries in $E$ are different and rearrange $A$ so the first three rows give:

$$
\left[\begin{array}{llllllll}
\ldots & 0 & 1 & 1 & 0 & 0 & 1 & \ldots \\
\ldots & 0 & 0 & 0 & 1 & 1 & 1 & \ldots \\
\ldots & a & 0 & 1 & b & c & a & \ldots
\end{array}\right]
$$

Because of the symmetry, we may assume the entry $a$ to be 0 . For $b$ and $c$ there are 3 cases to consider.

Case 1: $b=c=0$. Rows 2 and 3 have the configuration $\left[\begin{array}{lllll}0 & 1 & 1 & 1 & 0 \\ 0 & 0 & 0 & 0 & 1\end{array}\right]$ and therefore must not contain a configuration $\left[\begin{array}{l}1 \\ 1\end{array}\right]$. This forces all of the entries on the right for these 3 rows 
to be $\left[\begin{array}{l}1 \\ 1 \\ 0\end{array}\right]$ 's. If there are 2 or more of these, then rows 1 and 3 contain $\left[\begin{array}{lllll}0 & 1 & 1 & 1 & 1 \\ 0 & 0 & 0 & 0 & 1\end{array}\right]$. To avoid $F$ on rows 1 and 3 , there are at most two 1's in row 3 under the $\left[\begin{array}{l}0 \\ 0\end{array}\right]$ 's. Now we could delete the first 3 rows of $A$ and at most 7 columns ( the 4 columns of $E$, the common column $B_{2}$, and the columns with 1's in row 3) to get a simple matrix and so

$$
\operatorname{forb}(m, F) \leq \operatorname{forb}(m-3, F)+7
$$

and so the bound is proven.

Case 2: Assume $b=c=1$. But now the pairs of rows 1,3 and 2,3 both contain $K_{2}$ and this contradicts the choice of $C$.

Case 3: Assume $b=0, c=1$. In the first three rows we have

$$
\left[\begin{array}{llll}
1 & 1 & 0 & 0 \\
0 & 0 & 1 & 1 \\
0 & 1 & 0 & 1
\end{array}\right] .
$$

and so the pairs of rows 1,3 and 2,3 both contain $K_{2}$, contradicting the choice of $C$.

Corollary 2.7 For any submatrix of $F_{s}$ of $F=\left[\begin{array}{llllll}0 & 1 & 1 & 1 & 0 & 1 \\ 0 & 0 & 0 & 0 & 1 & 1\end{array}\right]$ containing either $F_{1}=\left[\begin{array}{llll}0 & 1 & 1 & 1 \\ 0 & 0 & 0 & 0\end{array}\right]$ or $F_{2}=\left[\begin{array}{llll}0 & 1 & 1 & 1 \\ 1 & 0 & 0 & 0\end{array}\right]$ and $m \geq 3$

$$
\operatorname{forb}\left(m, F_{s}\right)=\left\lfloor\frac{7}{3} m\right\rfloor+1 .
$$

Proof: The matrices $A_{m}$ constructed above do not contain the configuration $F_{2}$. We modify this construction slightly, setting $A_{3}^{\prime}=A_{3}, A_{4}^{\prime}=A_{4}, A_{5}^{\prime}=A_{5}$ and

$$
A_{m}^{\prime}=\left[\begin{array}{cccccccc}
A_{m-3}^{\prime} & \multicolumn{1}{c}{1^{\prime} \mathrm{s}} & & \\
& 0 & 0 & 0 & 1 & 1 & 1 & 1 \\
1 ' s & 0 & 1 & 1 & 0 & 0 & 1 & 1 \\
& 1 & 0 & 1 & 0 & 1 & 0 & 1
\end{array}\right]
$$

These matrices do not contain $F_{1}$.

The following exact bound uses graph theory to aid the analysis.

Theorem 2.8 For $F=\left[\begin{array}{lllll}1 & 1 & 0 & 0 & 0 \\ 0 & 0 & 1 & 1 & 1\end{array}\right]$ and $m \geq 3$,

$$
f o r b(m, F)=\left\lfloor\frac{8}{3} m\right\rfloor .
$$


Proof: We provide a construction for $A_{m}$, a simple matrix $m \times\left\lfloor\frac{8}{3} m\right\rfloor$ which avoids the configuration $F$. We take $A_{1}=K_{1}, A_{3}=K_{3}$ and

$$
A_{5}=\left[\begin{array}{llllll}
A_{3} & 0 & 0 & 1 & 1 & 1 \\
& 0 & 0 & 1 & 1 & 1 \\
0^{\prime} s & 1 & 0 & 1 & 0 & 1 \\
& 0 & 1 & 0 & 1 & 1
\end{array}\right]
$$

For $m \neq 1,2,3,5$, we can construct $A_{m}$ inductively using

$$
A_{m}=\left[\begin{array}{lcccccccc} 
& 0 & 1 & 1 & 1 & 1 & 1 & 1 & 1 \\
A_{m-3} & \vdots & \vdots & \vdots & \vdots & \vdots & \vdots & \vdots & \vdots \\
& 0 & 1 & 1 & 1 & 1 & 1 & 1 & 1 \\
& 1 & 1 & 1 & 1 & 0 & 0 & 0 & 0 \\
0 ' \mathrm{~s} & 1 & 1 & 0 & 0 & 1 & 1 & 0 & 0 \\
& 1 & 0 & 1 & 0 & 1 & 0 & 1 & 0
\end{array}\right] .
$$

Hence, for $m \geq 3$, we have forb $(m, F) \geq\left\lfloor\frac{8}{3} m\right\rfloor$. We now show the reverse inequality.

Assume the theorem true where the number of rows is strictly between 2 and $m$. Let $A$ be a simple matrix of dimensions $m \times \operatorname{forb}(m, F)$ with $m>3$. We construct a graph considering rows as vertices and having directed edges $i \rightarrow j$ if the number of [ $\left[\begin{array}{l}0 \\ 1\end{array}\right]$ 's in $\left[\begin{array}{l}R_{i} \\ R_{j}\end{array}\right]$ $<2$ and edges $i \cdots \cdots j$ if the numbers of [ $\left[\begin{array}{l}0 \\ 1\end{array}\right]$ 's and $\left[\begin{array}{l}1 \\ 0\end{array}\right]$ 's in $\left[\begin{array}{l}R_{i} \\ R_{j}\end{array}\right]$ are each $<3$. We then have the following properties.

(i) Each pair of rows is connected by at least one edge. This is clear.

(ii) The graph on the directed edges is transitive and contains no cycles. If $i \rightarrow j$ and $j \rightarrow k$, then we have the three possibilities<smiles></smiles>

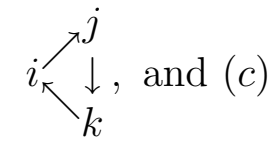<smiles>[Al]=[IH]</smiles>

For cases (a) and (b) we look at the possible entries for these three rows. The entries above the braces indicate the number of possible columns of these types.

$$
\begin{aligned}
& i \overbrace{0 \cdots 00 \cdots 01 \cdots 10 \cdots 0}^{\leq 1} \overbrace{1 \cdots 11 \cdots 1}^{\leq 1} 0 \cdots 01 \cdots 1 \\
& \text { j } 1 \cdots 11 \cdots 10 \cdots 00 \cdots 00 \cdots 01 \cdots 11 \cdots 11 \cdots 1 \text {. } \\
& k 0 \cdots 01 \cdots 10 \cdots 01 \cdots 10 \cdots 00 \cdots 00 \cdots 01 \cdots 1
\end{aligned}
$$

We can eliminate rows $i$ and $j$ and at most 4 columns to produce a simple matrix $A_{m-2}$. Using the second construction we produce an $m$ rowed simple matrix which does not 
contain $F$ but has more columns that $A$.

For $A$ to be maximal we can have (c) only - the graph must be transitive and, as well, there are no cycles.

(iii) Induction holds if …. edges occur other than in cliques.

If there are edges $i_{\ddots_{\ldots}} \nearrow_{j}^{j}$ then the possible entries for these rows are

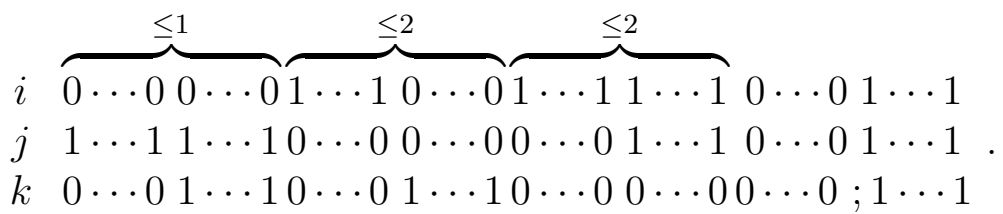

We eliminate rows $j$ and $k$ and at most 5 columns to produce a simple matrix $A_{m-2}$. But then

$$
|A| \leq\left|A_{m-2}\right|+5 \leq\left\lfloor\frac{8}{3}(m-2)\right\rfloor+5 \leq\left\lfloor\frac{8}{3} m\right\rfloor .
$$

(iv) The theorem is true if the … edges occur only in cliques.

Consider a clique with $k$ vertices. We wish to discover how many columns are possible which are not constant on these $k$ rows.

For $k=1$ there are no columns which are non-constant on this clique.

For $k>1$, the presence of the $\cdots . .$. edges means we avoid the configuration $\left[\begin{array}{lll}0 & 0 & 0 \\ 1 & 1 & 1\end{array}\right]$ in the clique. If a column has both 0 and 1 entries on these $k$ rows, it has at least $k-1$ of the $\left[\begin{array}{l}0 \\ 1\end{array}\right]$ configurations in these $k$ rows. More that $2 k$ of such columns would give more than $2 k(k-1)$ of the $\left[\begin{array}{l}0 \\ 1\end{array}\right]$ configurations in $k(k-1) / 2$ pairs of rows from this clique. One pair would then contain more than 4 and hence the configuration $\left[\begin{array}{lll}0 & 0 & 0 \\ 1 & 1 & 1\end{array}\right]$. Thus there can be no more than $2 k$ columns which are not constant on these $k$ rows. Let $c_{k}$ be the number of cliques which have $k$ vertices. The maximum number of columns of $A$ which are not constant on cliques is then $2\left(m-c_{1}\right)$.

There is an ordering on the cliques by defining $C_{1}<C_{2}$ if there is a directed edge $i \rightarrow j$ for $i \in C_{1}$ and $j \in C_{2}$. By properties (i) and (ii) this ordering is strictly linear. Reorder the rows of $A$ to respect this linear order. The columns of $A$ which are constant on cliques avoid the submatrix $\left[\begin{array}{ll}0 & 0 \\ 1 & 1\end{array}\right]$. If the number of cliques is $m^{\prime}$, then this is equivalent to avoiding this submatrix in an $m^{\prime}$ rowed matrix. There are a maximum of $m^{\prime}-1$ distinct columns possible which contain a $\left[\begin{array}{l}0 \\ 1\end{array}\right]$ and a maximum of $m^{\prime}+1$ columns with no $\left[\begin{array}{l}0 \\ 1\end{array}\right]$.

Thus, $A$ has a maximum of $2\left(m-c_{1}\right)+2 m^{\prime}$ columns, but where there are cliques of size 2 , we have overcounted. This is because on clique $C_{t}$ of size two, say on rows $i, i+1$, we can distinguish only 2 nonconstant columns. To achieve the count of 4 we must have $\left[\begin{array}{llll}0 & 0 & 1 & 1 \\ 1 & 1 & 0 & 0\end{array}\right]$ in rows $i, i+1$. Two of these columns could have 1's above and 0's below. The remaining two columns must either have 0's on a clique above $C_{t}$ and then a 1 in 
$C_{t}$ or a 1 's on a clique following $C_{t}$ and a 0 in $C_{t}$. The count of $m^{\prime}-1$ distinct columns possible which contain a $\left[\begin{array}{l}0 \\ 1\end{array}\right]$ must be reduced by at least 1 for every clique of size 2 (the two columns can both contribute a $\left[\begin{array}{l}0 \\ 1\end{array}\right]$ to the same clique pair).

We then have

$$
\begin{aligned}
|A| \leq 2\left(m-c_{1}\right)+2 m^{\prime}-c_{2} & =2 c_{1}+5 c_{2}+\sum_{k>2}(2 k+2) c_{k} \\
& \leq\left\lfloor\frac{8}{3}\left(c_{1}+2 c_{2}+3 c_{3}+\ldots\right)\right\rfloor=\left\lfloor\frac{8}{3} m\right\rfloor .
\end{aligned}
$$

\section{Quadratic Bounds}

In [4], we classified which $2 \times l F$ give rise to quadratic bounds. We are able to substantially strengthen the bounds obtaining some exact bounds or bounds with correct quadratic terms. Graph theory is remarkably useful.

In the following, we investigate forb $(m, F)$ for

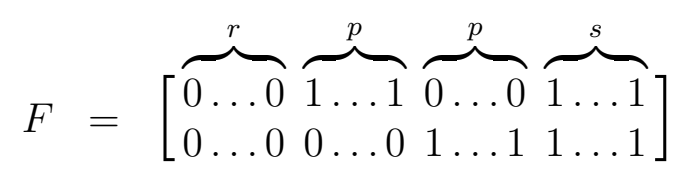

By symmetry we may assume $r \geq s \geq 1$. From [4], forb $(m, F)$ is quadratic if $r \geq 2$ or $r=s=1$ and $p \geq 2$. Certain general cases are known 'exactly'.

Theorem 3.1 ([3]) Let $0_{2, r}$ denote the $2 \times r$ matrix of 0 's. For $F$ as above with $r \geq 2, p, s$ or any submatrix $F_{s}$ of $F$ containing $0_{2, r}$, then $\operatorname{forb}(m, F)=\operatorname{forb}\left(m, F_{s}\right)=\operatorname{forb}\left(m, 0_{2, r}\right)=$ $\frac{r+1}{6} m^{2}+O(m)$.

In what follows we will often consider cases with $p>r$. We will assume $p \geq 2$. In [4]

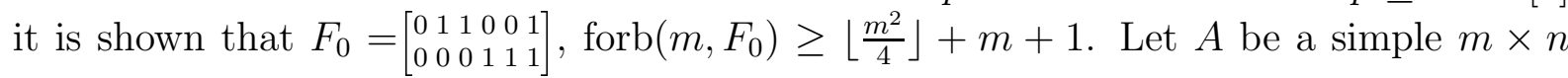
matrix which does not have the configuration $F$ of (2). Let $R_{i}$ refer to the $i$ th row of $A$. We consider the rows as vertices in a graph with the following edges:

(a) The directed edge $i \rightarrow j$ if the number of $\left[\begin{array}{l}0 \\ 1\end{array}\right]$ 's in $\left[\begin{array}{l}R_{i} \\ R_{j}\end{array}\right]$ is less than $p$.

(b) $i \cdots \cdots j$ if the number of $\left[\begin{array}{l}0 \\ 0\end{array}\right]$ 's in $\left[\begin{array}{l}R_{i} \\ R_{j}\end{array}\right]$ is less than $r$.

(c) $i \cdots \cdots j$ if the number of $\left[\begin{array}{l}1 \\ 1\end{array}\right]$ 's in $\left[\begin{array}{l}R_{i} \\ R_{j}\end{array}\right]$ is less than $s$.

We then have the following:

Lemma 3.2 If we are given forb $(m-1, F) \leq a(m-1)^{2}+b(m-1)+c$ (for $a, b>0$ and $a \leq b)$, then either forb $(m, F) \leq a m^{2}+b m+c$ or we may assume that for $m \geq$ $\frac{3}{2 a} \max \{p, r, s\}$ that each pair of rows of $A$ is connected by exactly one edge and, in the graph arising from $A$, the following 8 triangles do not occur: 


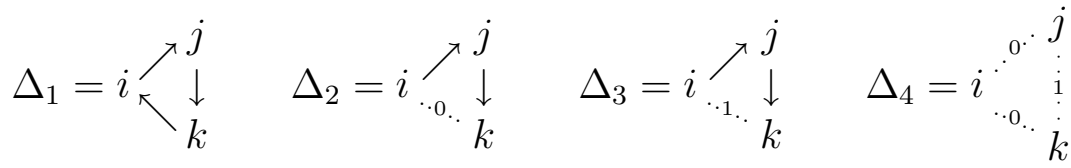

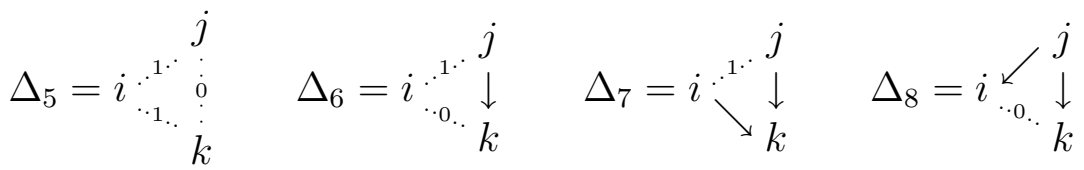

Proof: We compute $a m^{2}+b m+c-\left(a(m-1)^{2}+b(m-1)+c\right)=a(2 m-1)+b \geq 2 a m$. Thus we need only show that one of the cases of the Lemma yield

$$
\operatorname{forb}(m, F) \leq \operatorname{forb}(m-1, F)+2 a m
$$

(or forb $(m, F) \leq$ forb $(m-2, F)+2 a m$. Our choice of $m$ ensures that $3 \max \{p, r, s\} \leq 2 a m$ and this can be used to show the above inequality in what follows. In trying to prove forb $(m, F) \leq a m^{2}+b m+c$ by induction, the base cases can be handled with large $b$ and/or $c$. Since the results are not usually exact, this is reasonable.

For $A$ not to have the configuration $F$, each pair of rows must be connected by an edge. Since a directed edge may go either way there are $\left(\begin{array}{l}4 \\ 2\end{array}\right)=6$ possible combinations for rows $i$ and $j$ to be connected by two edges.

If, for example, we have the edges $i \rightarrow j$ and $i \cdots \cdots j$, then $A$ has the configuration

$$
\begin{aligned}
& R_{i} \overbrace{0 \ldots 0}^{\leq p-1} \overbrace{0 \ldots 0}^{\leq r-1} 1 \ldots 11 \ldots 1 \\
& R_{j} \quad 1 \ldots 10 \ldots 0001 \ldots 1 \text {. }
\end{aligned}
$$

Eliminating the first $\leq p+r-2$ columns and $R_{i}$ leaves a simple matrix, giving that

$$
\operatorname{forb}(m, F) \leq \operatorname{forb}(m-1, F)+p+r-2 .
$$

Using $p+r-2<2 a m$ would enable us to prove forb $(m, F) \leq a m^{2}+b m+c$ by induction. The proofs for the other 5 cases are similar.

For $\Delta_{1}$, we deduce

$$
\begin{aligned}
& R_{i} \overbrace{0 \ldots 00 \ldots 0}^{\leq p-1} \overbrace{0 \ldots 01 \ldots 1}^{\leq p-1} \overbrace{1 \ldots 11 \ldots 1}^{\leq p-1} 0 \ldots 01 \ldots 1 \\
& R_{j} \quad 1 \ldots 11 \ldots 10 \ldots 00 \ldots 00 \ldots 01 \ldots 10 \ldots 01 \ldots 1 \\
& R_{k} \quad 0 \ldots 01 \ldots 11 \ldots 11 \ldots 10 \ldots 00 \ldots 0001 \ldots 1
\end{aligned}
$$

Eliminating the first $\leq 3 p-3$ columns, $R_{i}$ leaves a simple matrix, so that $\operatorname{forb}(m, F) \leq$ 
forb $(m-1, F)+3 p-3$. For $\Delta_{2}$ we get

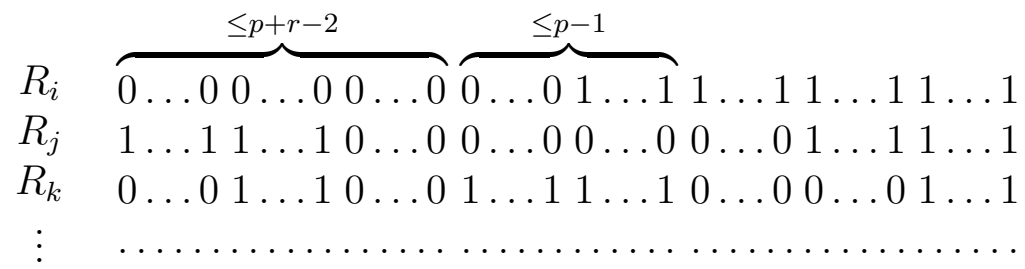

Eliminating the first $\leq 2 p+r-3$ columns and $R_{i}$ leaves a simple matrix, $\operatorname{so}$ forb $(m, F) \leq$ forb $(m-1, F)+2 p+r-3$. Symmetrically we get forb $(m, F) \leq \operatorname{forb}(m-1, F)+2 p+s-3$ with the triangle $\Delta_{3}$. For $\Delta_{4}$ we have

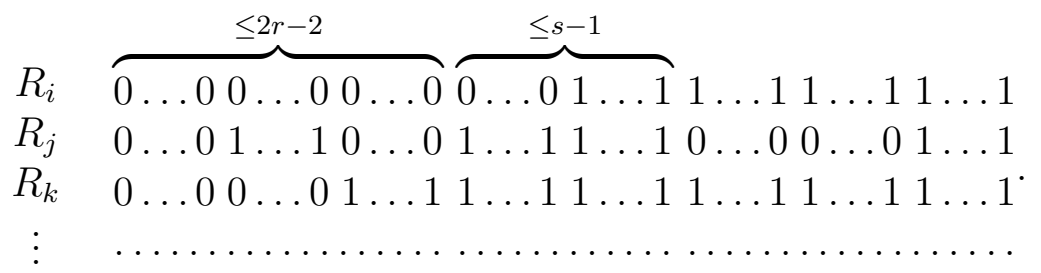

Eliminating row $i$ and the first set of $\leq 2 r+s-3$ columns leaves a simple matrix, so that $\operatorname{forb}(m, F) \leq \operatorname{forb}(m-1, F)+2 r+s-3$ Symmetrically we get forb $(m, F) \leq$ forb $(m-1, F)+2 s+r-3$. with the triangle $\Delta_{5}$. For $\Delta_{6}$ we have

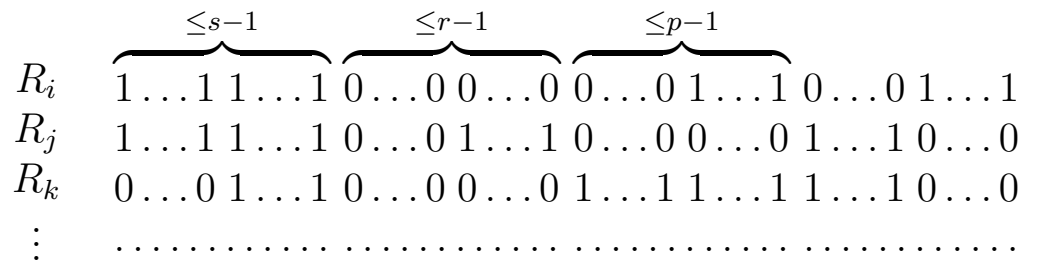

Eliminating the first $\leq p+r+s-3$ columns and $R_{j}$ leaves a simple matrix, so that forb $(m, F) \leq$ forb $(m-1, F)+p+r+s-3$. For $\Delta_{7}$ we have

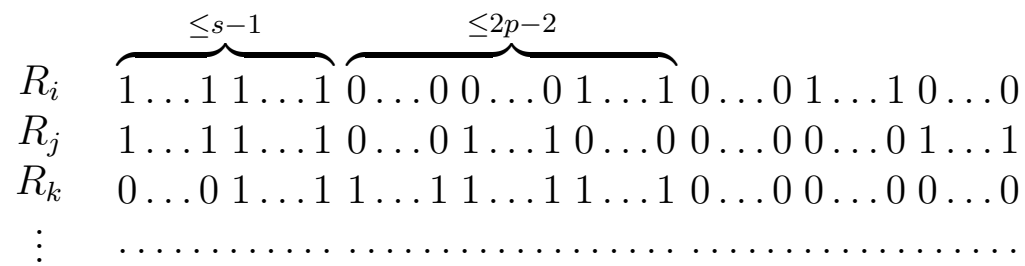

Eliminating the first $\leq p+r+s-3$ columns and $R_{k}$ leaves a simple matrix, so that forb $(m, F) \leq \operatorname{forb}(m-1, F)+2 p+s-3$. Symmetrically we get forb $(m, F) \leq \operatorname{forb}(m-$ $1, F)+2 p+r-3$ with the triangle $\Delta_{8}$.

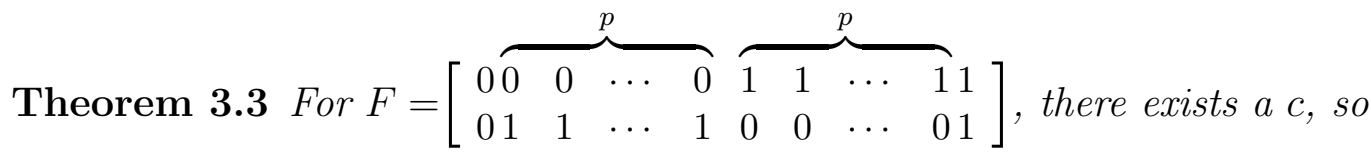

$$
\frac{m^{2}}{4}+\left(p-1 \frac{1}{2}-\sqrt{p-1}\right) m+O(p) \leq f o r b(m, F) \leq \frac{m^{2}}{4}+(p-1)(m-2)+c
$$


where the lower bound holds for $p \geq 4$ and $m>4(p-1)^{3 / 2}$.

In addition, there exists an $m_{0}$ so that for $m>m_{0}$ we may take $c=0$ in the upper bound.

Proof: Let $A$ be an $m$ rowed matrix with no configuration $F$. Form a graph on the rows of $A$ as above $(r=s=1)$ with edges $i \rightarrow j, i \cdots \cdots j, i \cdots \cdots j$. To prove the upper bound we use induction on $m$ with $m \geq 6 p$. We can choose $c$ large enough ( $2^{6 p}$ will do) so that $\operatorname{forb}(m-1, F) \leq \frac{(m-1)^{2}}{4}+(p-1)((m-1)-2)+c$. Applying Lemma 3.2, (using $\left.6 p \geq \frac{3}{2 a} \max \{p, r, s\}\right)$ we may assume $A$ has no doubled edges nor the forbidden triangles. Property 1: If we have $i \rightarrow j$ and $j \rightarrow k$, then $i \rightarrow k$ (transitivity). This follows from the three forbidden triangles $\Delta_{1}, \Delta_{2}, \Delta_{3}$.

Property 2: If $i \rightarrow k$ and $j \rightarrow k$ and yet $i, j$ are not joined by a directed edge then there is an edge $i \cdots \cdots j$. This follows from the forbidden triangle $\Delta_{7}$.

Property 3: If $i \rightarrow j$ and $i \rightarrow k$ and yet $j, k$ are not joined then there is an edge $j \cdots \cdots k$. This follows from the forbidden triangle $\Delta_{8}$.

Property 4: If $i \rightarrow j$ and $j \cdots \cdots * k$ then $i \cdots 0 \cdots k$. We need the forbidden triangles $\Delta_{2}, \Delta_{6}, \Delta_{8}$. Property 5: Symmetrically if $i \rightarrow j$ and $i \cdots \cdots k$ then $j \cdots 1 \cdots k$. We need the forbidden triangles $\Delta_{3}, \Delta_{6}, \Delta_{7}$

We decompose the rows into two sets $M_{1}, M_{2}$ as follows. Identify a maximal clique $C_{1}$ of at least two rows such that every pair $i, j \in C_{1}$ are joined $i \cdots \cdots j$. Such a clique exists since otherwise our matrix $A$ is avoiding the configuration $F^{\prime}$ obtained from $F$ by deleting the column of 0 's but that yields a linear bound of $(p-1) m+2$. In addition assume $C_{1}$ has the property that there is no clique $C^{\prime}$ below $C_{1}$, namely no clique $C^{\prime}$ of (at least two) rows such that every pair $i, j \in C^{\prime}$ are joined $i \cdots \cdots j$ and for every $k \in C$ there is a $l \in C^{\prime}$ so that there is a directed walk from $k$ to $l$. Let $M_{1}$ be the rows $i$ for which there exists a $k \in C$ with a directed path from $i$ to $k$. We deduce by properties 1,2,4,5 that there are no edges $i \cdots 1 . j$ for $i, j \in M_{1}$. Use the techniques of Lemma 2.1 with the edges .... being considered non edges, to find a forest of indirected trees $T_{1}$ on the rows $M_{1}$ and a row ordering. Alternatively, we can obtain an acyclic ordering of $M_{1}$ since there are no directed cycles (because of the transitivity and lack of 2-cycles by Lemma 3.2). Then delete the edges implied by transitivity to obtain $T_{1}$, a forest of indirected trees whose roots are $C_{1}$.

Let $M_{2}$ be the remaining rows of $A$. We assert that for no edges $k \cdots \cdots l$ for $k, l \in M_{2}$. Assume the contrary that $k \cdots \cdot \cdot l$ exists. Then by the definition of $M_{1}$, there are no edges from $k$ or $l$ to any $i \in C_{1}$. Let $D \subseteq C_{1}$ be the $i \in C_{1}$ for which there is either an edge $i \rightarrow k$ or $i \rightarrow l$. Let $E \subseteq C_{1}$ be the $i \in C_{1}$ for which there is either an edge $i \cdots \cdots k$ and $i \cdots 0 \cdots l$. If $D \cup E=C_{1}$ then this would contradict the choice of $C_{1}$ with $C^{\prime}=E \cup\{k, l\}$ as a clique below $C_{1}$. Thus for some $i \in C_{1}$, we have $i \cdots 1 \cdots k$ and $i \cdots \cdots l$ but then this is a forbidden triangle $\Delta_{8}$ or we have $i \cdots 1 \cdots k$ and $i \cdots \cdot \cdots l$ (or vice versa) but then this is a forbidden triangle $\Delta_{7}$. We conclude that there are no edges $k \cdots \cdots l$ for $k, l \in M_{2}$. As above, use Lemma 2.1 with $\cdots \cdots$ edges being considered non edges to form a forest of outdirected trees $T_{2}$ on the rows $M_{2}$.

Let $\left|M_{1}\right|=m_{1},\left|M_{2}\right|=m_{2}$. Using the methods of Theorem 2.2, there are at most 
$m_{1}+1$ columns on rows $M_{1}$, which do not have a $\left[\begin{array}{l}0 \\ 1\end{array}\right]$ on an edge of $T_{1}$. Similarly, there are at most $m_{2}+1$ colums on rows $M_{2}$ which do not have $\left[\begin{array}{l}0 \\ 1\end{array}\right]$ on an edge of $T_{2}$. Hence at most $\left(m_{1}+1\right)\left(m_{2}+1\right)$ columns in $A$ do not have a $\left[\begin{array}{l}0 \\ 1\end{array}\right]$ on an edge of $T_{1} \cup T_{2}$. All other columns of $A$ have a $\left[\begin{array}{l}0 \\ 1\end{array}\right]$ on an edge of $T_{1}$ or $T_{2}$ and so by the standard pidgeonhole argument there are at most $(p-1)(m-2)$ such columns. We may maximize this bound $\left(m_{1}+1\right)\left(m_{2}+1\right)+(p-1)(m-2)$ by taking $m_{1}=\lfloor m / 2\rfloor, m_{2}=\lceil m / 2\rceil$ and we get the bound.

To reduce $c$ to 0 for large $m$, consider $A=A_{0}$ as an $m \times n$ simple matrix with no configuration $F$. If $A_{0}$ has no triangle or doubled edge then our above argument gives the bound with $c=0$. Assume, $A_{0}$ has a triangle or doubled edge. Using the arguments of Lemma 3.2 we may delete one row and some columns to obtain an $(m-1) \times n_{1}$ simple matrix $A_{1}$ with no configuration $F$ and $n_{1}>n-3 p$. Now if $A_{1}$ has no triangle or doubled edge, then $n_{1} \leq \frac{(m-1)^{2}}{4}+(p-1)((m-1)-2)$ and then, for $m \geq 6 p$ we have $n \leq \frac{m^{2}}{4}+(p-1)(m-2)$. If $A_{1}$ has a triangle or a doubled edge, then we can delete one row and some columns to obtain a simple $(m-2) \times n_{2}$ matrix $A_{2}$, and we repeat as for $A_{1}$. Either we will have shown the bound for $m$ with $c=0$ or we will obtain an $(m-k) \times n_{k}$ simple matrix $A_{k}$ with no configuration $F$ and $n_{k}>n-3 k p$. Assume $n>\frac{m^{2}}{4}+(p-1)(m-2)$. We have for $A_{k}$ that $n-3 k p<n_{k} \leq \frac{(m-k)^{2}}{4}+(p-1)((m-k)-2)+c$ by induction. We combine to get

$$
m<2\left(2 p+1+\frac{c}{k}+\frac{k}{4}\right)
$$

Taking $k=2 \sqrt{c}$ we get $m<2(\sqrt{c}+2 p+1)=m_{0}$. Hence we can assume $c=0$ for $m \geq m_{0}$.

We construct a simple $m$-rowed matrix avoiding $F$ as follows. Let $m_{1}=\left\lfloor\frac{m}{2}\right\rfloor, m_{2}=$ $m-m_{1}, l_{1}=\lfloor\sqrt{p-1}\rfloor, l_{2}=\left\lfloor\frac{p-1}{l_{1}}\right\rfloor$. The graph on the upper $m_{1}$ rows of $A$ where we eliminate directed edges implied by transitivity will consist of disjoint paths of length $l_{1}$ (and one of $r_{1}$ ) and on the lower $m_{2}$ rows, disjoint paths of length $l_{2}$ (and one of $r_{2}$ ). Define the $l \times l$ matrices

$$
K_{1, l}=\left[\begin{array}{cccc}
0 & & & \\
& 0 & 1 ' s & \\
& 0 ' s & \ddots & \\
& & & 0
\end{array}\right] \text { and } K_{2, l}=\left[\begin{array}{cccc}
1 & & & \\
& 1 & 1 ' s & \\
& 0 \text { 's } & \ddots & \\
& & & 1
\end{array}\right]
$$

There are no $\left[\begin{array}{l}0 \\ 1\end{array}\right]$ submatrices in $K_{1, l}$ and $K_{2, l}$. There are no $\rightarrow$ edges between upper and lower rows of these matrices. We use these blocks to obtain the $m_{1} \times\left(m_{1}+1\right)$ and 
$m_{2} \times\left(m_{2}+1\right)$ matrices

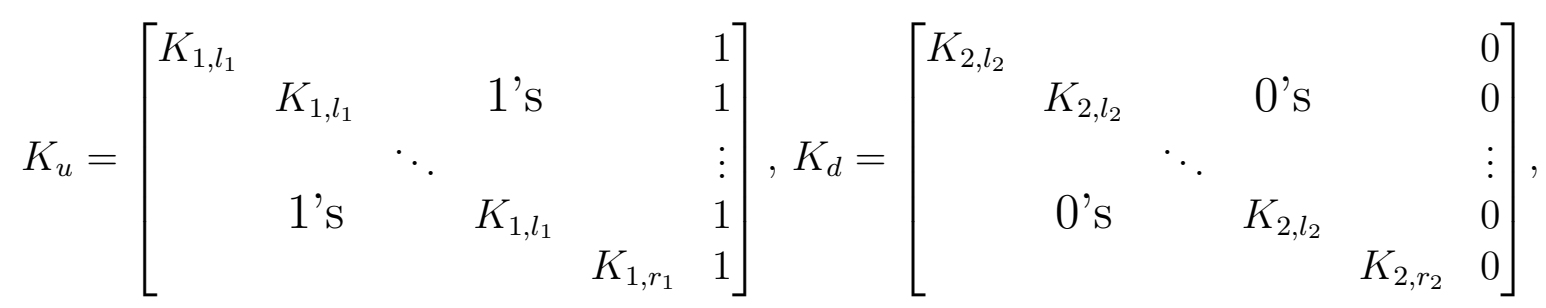

where $r_{1}$ and $r_{2}$ are the integer remainders in dividing $m_{1}$ and $m_{2}$ by $l_{1}$ and $l_{2}$ respectively. Note that $K_{u}$ has no $\left[\begin{array}{l}0 \\ 0\end{array}\right]$ and $K_{u}$ has no $\left[\begin{array}{l}1 \\ 1\end{array}\right]$

In the product

$$
A_{1}=K_{u} \otimes K_{d}
$$

we have $\rightarrow$ trees of length $l_{1}$ on top and trees of length $l_{2}$. The bottom rows of the $K_{1, l}$ blocks on top and the top rows of the $K_{2, l}$ blocks on the bottom become the roots of paths. The maximum number of [ $\left[\begin{array}{l}0 \\ 1\end{array}\right]$ 's in a pair of rows $\left[\begin{array}{l}R_{i} \\ R_{j}\end{array}\right]$ is $l_{1} l_{2}<p-1$ occurring when both $i$ and $j$ are roots of paths. When $i$ and $j$ are not roots the maximum number is $\max \left\{\left(l_{1}-1\right) l_{2}, l_{1}\left(l_{2}-1\right)\right\} \leq p-3$ when $p>4$.

Between two rows $i<j$ in the same path in the top (or in the bottom) there are no $\left[\begin{array}{l}0 \\ 1\end{array}\right]$ 's in $\left[\begin{array}{l}R_{i} \\ R_{j}\end{array}\right]$. For each edge $i \rightarrow(i+1)$ in these $\left\lceil\frac{m_{1}}{l_{1}}\right\rceil+\left\lceil\frac{m_{2}}{l_{2}}\right\rceil$ trees, we add $p-1$ columns to our matrix to produce the matrix $A$ so that

$$
\begin{aligned}
|A| & =\left(m_{1}+1\right)\left(m_{2}+1\right)+(p-1)\left(m-\left\lceil\frac{m_{1}}{l_{1}}\right\rceil-\left\lceil\frac{m_{2}}{l_{2}}\right\rceil\right) \\
& \geq \frac{m^{2}}{4}+\left(p-1-\sqrt{p-1}-\frac{1}{2}\right) m+O(p),
\end{aligned}
$$

since

$$
\begin{aligned}
(p-1)\left(\left\lceil\frac{m_{1}}{l_{1}}\right\rceil+\left\lceil\frac{m_{2}}{l_{2}}\right\rceil\right) & \leq \frac{p-1}{l_{1}} m_{1}+\frac{p-1}{l_{2}} m_{2}+2(p-1) \\
& \leq\left(l_{1}+2\right) m_{1}+(\sqrt{p-1}) m_{2}+2(p-1) \\
& \leq(\sqrt{p-1}+1) m+2(p-1) .
\end{aligned}
$$

Let $U_{1}, U_{2}$ be $m_{1}$ rowed matrices consisting of columns with a single $\mathrm{O}$. $U_{1}$ includes the $m_{1}-\left\lceil\frac{m_{1}}{l_{1}}\right\rceil$ columns not in $K_{u}$. $U_{2}$ consists of $p-1$ such columns chosen from $K_{u}$ (here we need $\left.m>4(p-1)^{3 / 2}\right)$. Similarly let $D_{1}, D_{2}$ be the corresponding $m_{2}$ rowed matrices of columns with a single 1 with $D_{1}$ being from those not in $K_{d}$ and $D_{2}$ being $p-1$ chosen from $K_{d}$. We then have

$$
A=\left[A, U_{1} \otimes D_{2}, U_{2} \otimes D_{1}\right]
$$

With $p>4$ and $m>4(p-1)^{3 / 2},\left\lceil\frac{m_{2}}{l_{2}}\right\rceil>\frac{m}{2(\sqrt{p-1}+2)} \geq p-1$, so that we obtain enough columns from $K_{u}$ and $K_{d}$. We add no more that two $\left[\begin{array}{l}0 \\ 1\end{array}\right]$ 's to each $\left[\begin{array}{l}R_{i} \\ R_{j}\end{array}\right]$, where $i, j$ are 
rows from the top and bottom, respectively, without both being roots. The count never exceeds $p-3+2=p-1$.

An exact bound is possible for $p=2$, improving on a result in [4].

Theorem 3.4 For $F=\left[\begin{array}{llllll}0 & 0 & 0 & 1 & 1 & 1 \\ 0 & 1 & 1 & 0 & 0 & 1\end{array}\right]$

$$
\operatorname{forb}(m, F)=\left\lfloor\frac{m^{2}}{4}\right\rfloor+m+1
$$

Proof: The construction can be found as Configuration 5 in [4]. We can tighten the proof found there by seeking copies of $K_{2}$. Let $A$ be an $m \times\left(\left\lfloor\frac{m^{2}}{4}\right\rfloor+m+2\right)$ simple matrix with no $F$. Since forb $\left(m, K_{2}\right)=m+1$, on the first $m+2$ columns we can find a copy of $K_{2}$, say on rows $i, j$, and deduce that either $\left.\begin{array}{l}i \\ j\end{array}\right]$ or ${ }_{j}^{i}\left[\begin{array}{l}1 \\ 0\end{array}\right]$ occurs just once in $A$ in rows $i, j$. Set aside the column of $A$ that that has the single occurence and repeat on the remaining columns which can be done if there are at least $m+2$ columns left. Note that we can no longer find $K_{2}$ on rows $i, j$ after the deletion. We will have set aside $\left\lfloor\frac{m^{2}}{4}\right\rfloor+1$ columns. Form a graph $G$ on the rows by joining $i, j$ if we selected a $K_{2}$ on those rows. Thus $G$ has $\left\lfloor\frac{m^{2}}{4}\right\rfloor+1$ edges. Following the proof in [4] we can verify that the graph has no triangles. By Turán's bound, the number of edges is at most $\left\lfloor\frac{m^{2}}{4}\right\rfloor$ and this contradiction proves the bound.

The following result shows some promise for obtaining exact bounds for all $2 \times l F$.

Theorem 3.5 Let

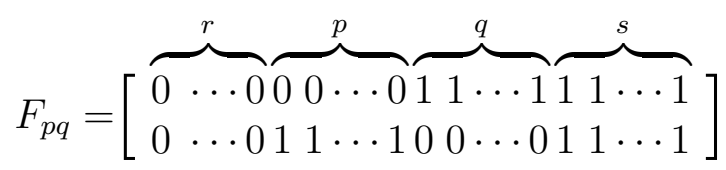

with $p<q$. If forb $\left(m, F_{p p}\right) \leq a m^{2}+b m+c$ for $0<a, b$, then for $m>q / a+a / 2$, there exists a $c^{\prime} \geq c$ so that

$$
\operatorname{forb}\left(m, F_{p q}\right) \leq a m^{2}+b m+c^{\prime} .
$$

There exists an $m_{0}$ so that for $m>m_{0}$ we can replace $c^{\prime}$ by $c$.

Proof: Following in the spirit of Lemma 3.2 we show that forb $\left(m, F_{p q}\right) \leq a m^{2}+b m+c^{\prime}$ by induction on $m$. We establish the base cases for $m \leq q / a+a / 2$ by choosing $c^{\prime}$ large enough. If $A$ is a simple $m \times a m^{2}+b m+c^{\prime}+1$ matrix with no $F_{p q}$, then $A$ contains a configuration $F_{p p}$ say on rows 1,2 . We deduce that $A$ has at most $2(q-1)$ columns with the configuration $\left[\begin{array}{l}0 \\ 1\end{array}\right]$ in rows 1,2 and so we could delete row 1 and at most $2(q-1)$ columns to obtain a simple matrix $A^{\prime}$ with no $F_{p q}$. Applying induction and the fact that 
$a m^{2}+b m+c^{\prime}-\left(a(m-1)^{2}+b(m-1)+c^{\prime}>2(q-1)\right.$ for $m>q / a+a / 2$, we deduce that $A^{\prime}$ has $F_{p q}$, a contradiction. Thus for all $m$

$$
\operatorname{forb}\left(m, F_{p q}\right) \leq a m^{2}+b m+c^{\prime}
$$

To establish $c^{\prime}=c$ for $m$ large, we assume $c^{\prime}>c$ and let $A$ be an $m \times a m^{2}+b m+c+1$ simple matrix with no $F_{p q}$. Thus $A$ contains $F_{p p}$, say on rows 1,2 . As above, we can delete row 1 and at most $2(q-1)$ columns to obtain a simple matrix $A^{\prime}$. But, $a m^{2}+b m+c+1-2(q-1)>$ $a(m-1)^{2}+b(m-1)+c^{\prime}$ for $m>\left(2 q-3+a^{2}+c^{\prime}-c-b\right) / 2 a=m_{0}$. Hence for $m>m_{0}$, $A^{\prime}$ has $F_{p q}$, a contradiction. Hence we may choose $c^{\prime}=c$ for $m>m_{0}$.

Theorem 3.6 Let

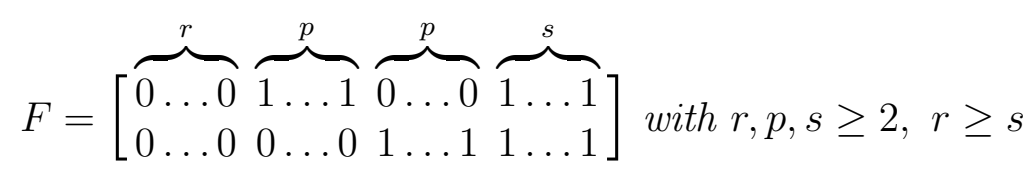

then forb $(m, F) \leq(r-1) m^{2} / 2+O(m)$. Moreover for $m>p$ and $r, s \geq 3$, we have $\lim _{p \rightarrow \infty} \operatorname{forb}(m, F) / m^{2}=(r-1) / 2$.

Proof: We use induction on $r$. We first consider the case $r=2$ and follow the proof technique for Theorem 3.3. Let $A$ be a matrix with no configuration $F$. Obtain the decomposition $M_{1}$ and $M_{2}$ as well as forests $T_{1}$ and $T_{2}$. As before, with $\left|M_{1}\right|=m_{1}$, $\left|M_{2}\right|=m_{2}$, we find that there are at most $\left(m_{1}+1\right)\left(m_{2}+1\right)$ columns with no ${ }_{j}^{i}\left[\begin{array}{l}0 \\ 1\end{array}\right]$ on an edge $i \rightarrow j$ of $T_{1}$ or $T_{2}$, or $\left[\begin{array}{l}0 \\ 0\end{array}\right]$ on an edge $i \cdots \cdots \cdots j$ with $i, j \in M_{1}$ or $\left[\begin{array}{l}1 \\ 1\end{array}\right]$ on an edge $i \cdots \cdots j$ with $i, j \in M_{2}$. There are at most $(p-1)\left(m_{1}-1+m_{2}-1\right)+\left(\begin{array}{c}m_{1} \\ 2\end{array}\right)+\left(\begin{array}{c}m_{2} \\ 2\end{array}\right)$ other columns before we violate the definition of our edges. Note that $\left(m_{1}+1\right)\left(m_{2}+1\right)+\left(\begin{array}{c}m_{1} \\ 2\end{array}\right)+\left(\begin{array}{c}m_{2} \\ 2\end{array}\right)=\frac{m^{2}}{2}+O(m)$ using $m_{1}+m_{2}=m$. The result now follows.

For general $r>3$, consider the smaller matrix

$$
F^{\prime}=\left[\begin{array}{llllll}
0 \ldots 0 & \overbrace{1 \ldots 1}^{r-1} & \overbrace{0 \ldots 0}^{p} & \overbrace{1 \ldots 1}^{r-1} \\
0 \ldots 0 & 0 \ldots 0 & 1 \ldots 1 & 1 \ldots 1
\end{array}\right] .
$$

In the first forb $\left(m, F^{\prime}\right)$ columns we can find a copy of $F^{\prime}$, say in rows $i, j$. Then there are either at most $r-1\left[\begin{array}{l}0 \\ 0\end{array}\right]$ 's or at most $r-1\left[\begin{array}{l}1 \\ 1\end{array}\right]$ 's in rows $i, j$. In the former case, remove a column with $\left[\begin{array}{l}0 \\ 0\end{array}\right]$ in rows $i, j$ and in the latter case a column with $\left[\begin{array}{l}1 \\ 1\end{array}\right]$. Now rows $i, j$ do not contain $F^{\prime}$. Thus after $\left(\begin{array}{c}m \\ 2\end{array}\right)$ deletions we must stop. Hence

$$
\operatorname{forb}(m, F) \leq \operatorname{forb}\left(m, F^{\prime}\right)+\left(\begin{array}{c}
m \\
2
\end{array}\right)
$$

The result follows by induction. 
A construction of an $m$-rowed matrix proves the second conclusion. We start with a division of the matrix into a set of $m_{1}$ rows on top and a disjoint set of $m_{2}$ rows on the bottom. We start with $\left[K_{m_{1}}^{m_{1}}, K_{m_{1}}^{m_{1}-1}\right] \otimes\left[K_{m_{2}}^{0}, K_{m_{2}}^{1}\right]$ and then add the columns $K_{m_{1}}^{m_{1}-2} \otimes K_{m_{2}}^{0}$ and $K_{m_{1}}^{m_{1}} \otimes K_{m_{2}}^{m_{2}-2}$ for a total of

$$
\left(m_{1}+1\right)\left(m_{2}+1\right)+\left(\begin{array}{c}
m_{1} \\
2
\end{array}\right)+\left(\begin{array}{c}
m_{2} \\
2
\end{array}\right)=\frac{m^{2}+m+2}{2}
$$

distinct columns. The trees at the top and bottom will consist of single vertices. At this stage there is a single $\left[\begin{array}{l}0 \\ 1\end{array}\right]$ in each pair of rows $\left[\begin{array}{l}R_{i} \\ R_{j}\end{array}\right]$ where $R_{i}$ is a row at the top, $R_{2}$ is a row at the bottom. We now add more columns containing $r-2$ copies of $K_{m_{1}}^{m_{1}-2}$ at the top but with entries at the bottom chosen from $I_{m_{2}}$ so the columns are distinct and we get no more than $p-1\left[\begin{array}{l}0 \\ 1\end{array}\right]$ 's in each pair of rows one from the top and one from the bottom. This gives $r-1\left[\begin{array}{l}0 \\ 0\end{array}\right]$ 's in each pair of rows from the top and hence avoids $F$.

For a column with 0's in the top rows $i$ and $j$ (in the top $m_{1}$ rows) in the $k$ th additional copy of $K_{m_{1}}^{m_{1}-2}$ we choose the bottom 0 's apart from a 1 in the $i+j+(k-1) m_{1}\left(\bmod m_{2}\right)$-th entry. With $m_{2}=\left[\frac{(r-2) m}{p-2}\right]$ and $m_{1}<m$, this ensures that we do not get more than $p-2$ additional $\left[\begin{array}{l}0 \\ 1\end{array}\right]$ 's between a row at the top and a row at the bottom.

We now add columns which have 1's in the top rows and different entries chosen from $K_{m_{2}}^{3}$ so that we add the maximum number of columns without obtaining more than $s-1$ $\left[\begin{array}{l}1 \\ 1\end{array}\right]$ 's in each pair of rows from the bottom. This is a design problem; the number of extra columns of this form is approximately $\frac{s-2}{3}\left(\begin{array}{c}m_{2} \\ 2\end{array}\right)$.

The number of columns in this matrix is then approximately

$$
\frac{m^{2}+m+2}{2}+(r-2)\left(\begin{array}{c}
m_{1} \\
2
\end{array}\right)+\frac{s-2}{3}\left(\begin{array}{c}
m_{2} \\
2
\end{array}\right) .
$$

As $p$ increases, $m_{1}=m-m_{2}=\left\lfloor\frac{(p-r) m}{p-2}\right\rfloor$ approaches $m$, so that the coefficient of $m^{2}$ in this expression approaches $(r-1) m^{2} / 2$.

\section{A new bound for copies of the identity}

For an $m \times n$ matrix $A$, let $t \cdot A$ denote the $m \times t n$ matrix obtained from $t$ copies of $A$ (this notation is ambiguous but in this paper we will not be multiplying a matrix by a constant). Let $I_{k}$ denote the $k \times k$ identity matrix. Before this we only had forb $\left(m, p \cdot I_{k}\right)$ to be $\mathrm{O}\left(m^{k}\right)$ by Theorem 1.1 .

Theorem 4.1 We have forb $\left(m, p \cdot I_{k}\right)=O\left(m^{k-1+\frac{1}{k}}\right)$ and forb $\left(m, p \cdot I_{k}\right)=\Omega\left(m^{k-1}\right)$.

Proof: We use induction on $p$ noting that Theorem 1.2 gives forb $\left(m, I_{k}\right)=O\left(m^{k-1}\right)$. Let $A$ be an $m \times n$ simple matrix with no configuration $p \cdot I_{k}$. Assume $n>\operatorname{forb}\left(m,(p-1) \cdot I_{k}\right)$ 
so that $A$ has $(p-1) \cdot I_{k}$ on rows $\left\{i_{1}, i_{2}, \ldots, i_{k}\right\}$. Since $A$ doesn't have $p \cdot I_{k}$, there is some column of $I_{k}$ occurring exactly $p-1$ times in $A$. Without loss of generality we may assume that the column has 0 's in rows $i_{1}, i_{2}, \ldots, i_{k-1}$ and a 1 in row $i_{k}$. We call the set of $p-1$ columns of $A$ with 0 's in rows $i_{1}, i_{2}, \ldots, i_{k-1}$ and a 1 in row $i_{k}$ to be a special set of columns on $\left\{i_{1}, i_{2}, \ldots, i_{k}\right\}$.

If we assume

$$
n>\operatorname{forb}\left(m,(p-1) \cdot I_{k}\right)+(p-1) m^{k-1+\frac{1}{k}}
$$

we can choose $m^{k-1+\frac{1}{k}}$ disjoint special sets of $p-1$ columns by removing them one set at a time. Use the notation $\left(\begin{array}{c}X \\ t\end{array}\right)$ to be all subsets of $X$ of size $t$. Let $[m]=\{1,2, \ldots, m\}$. For $S=\left\{i_{1}, i_{2}, \ldots i_{k-1}\right\} \subseteq\left(\begin{array}{c}{[m]} \\ k-1\end{array}\right)$, let $a_{S}$ denote the number of disjoint special sets of columns on rows $i_{1}, i_{2}, \ldots, i_{k-1}, j$ for any $j$ i.e. special sets with 0 's in rows of $S$. Thus

$$
\sum_{S \in\left(\begin{array}{l}
{[m]} \\
k-1
\end{array}\right)} a_{S} \geq m^{k-1+\frac{1}{k}}
$$

Given $a_{S}$ column disjoint special sets on rows $i_{1}, i_{2}, \ldots, i_{k-1}, j$, for $j \in\left\{j_{1}, j_{2}, \ldots, j_{a_{S}}\right\}$, then the columns will have 0 's in all rows $j \in\left\{j_{1}, j_{2}, \ldots, j_{a_{S}}\right\}$ apart from one row, because the special sets are disjoint. Thus we get $\left(\begin{array}{c}a_{S} \\ k\end{array}\right)$ copies of $(p-1) \cdot I_{k}$ inside those $(p-1) a_{S}$ columns and $a_{S}$ rows $\left\{j_{1}, j_{2}, \ldots, j_{a_{S}}\right\}$ and so we get $\left(\begin{array}{c}a_{S} \\ k\end{array}\right)$ new special sets columns contained in the $(p-1) a_{S}$ columns. Thus

$$
\sum_{S \in\left(\begin{array}{l}
{[m]} \\
k-1
\end{array}\right)}\left(a_{S}+\left(\begin{array}{c}
a_{S} \\
k
\end{array}\right)\right) \leq\left(\begin{array}{c}
m \\
k
\end{array}\right) .
$$

This yields a contradiction since the sum $\sum_{S \in\left(\begin{array}{c}{[m]} \\ k-1\end{array}\right)}\left(a_{S}+\left(\begin{array}{c}a_{S} \\ k\end{array}\right)\right)$ will ba minimized (by convexity) with $a_{S}=m^{k-1+\frac{1}{k}} /\left(\begin{array}{c}m \\ k\end{array}\right)$ and so $\left(\begin{array}{c}a_{S} \\ k\end{array}\right)>m$. Now $\sum_{S \in\left(\begin{array}{c}{[m]} \\ k-1\end{array}\right)}\left(\begin{array}{c}a_{S} \\ k\end{array}\right)>m\left(\begin{array}{c}m \\ k-1\end{array}\right)>\left(\begin{array}{c}m \\ k\end{array}\right)$. This is a contradiction and so $n \leq \operatorname{forb}\left(m,(p-1) \cdot I_{k}\right)+(p-1) m^{k-1+\frac{1}{k}}$. Use induction on $p$ to get $\operatorname{forb}\left(m, p \cdot I_{k}\right)=O\left(m^{k-1+\frac{1}{k}}\right)$.

A construction to show forb $\left(m, p \cdot I_{k}\right)=\Omega\left(m^{k-1}\right)$ is due to Füredi and Quinn [8].

We believe the truth is forb $(m, F)=\Theta\left(m^{k-1}\right)$.

\section{References}

[1] R.P. Anstee, General forbidden configuration theorems, J. Combin. Th. (A) 40, (1985), 108-124.

[2] R.P. Anstee And Z. Füredi, Forbidden Submatrices, Discrete Math. 62,(1986), 225-243.

[3] R.P. Anstee, A forbidden configuration theorem of Alon, J. Combin.Th. (A) 47(1988), 16-27. 
[4] R.P. Anstee, J.R. Griggs, and A. Sali, Small Forbidden Configurations, Graphs and Combin. 13(1997), 91-118.

[5] P. Erdős And Stone, On the structure of linear graphs, Bull. Amer. Math. Soc. 52,(1946), 1087-1091.

[6] P. Frankl, On the trace of finite sets, J. Combin. Th. (A) 34, (1983), 41-45.

[7] P. Frankl, Z. Füredi And J. Pach, Bounding One-Way Differences, Graphs and Combin. 3, (1987), 341-347.266

[8] Z. FÜRedi And F. Quinn, Traces of finite sets, Ars Combin. 18, (1983), 195-200.

[9] Z. FÜREDI, Private communication.

[10] N. Sauer, On the density of families of sets, J. Combin. Th. (A) 13(1973) 145-147.

[11] L. RÉDEI, Ein kombinatorischer Satz, Acta Litt. Szeged 7(1934),39-43.

[12] S. Shelah, A combinatorial problem: Stability and order for models and theories in infinitary languages, Pac. J. Math. 4(1972), 247-261.

[13] V.N. Vapnik And A.Ya. Chervonenkis, On the uniform convergence of relative frequencies of events to their probabilities, Th. Prob. and Applics. 16(1971), 264-280.

\section{Appendix: Some Basic Linear Bounds}

This appendix contains some new basic bounds for completeness. Careful counting is employed.

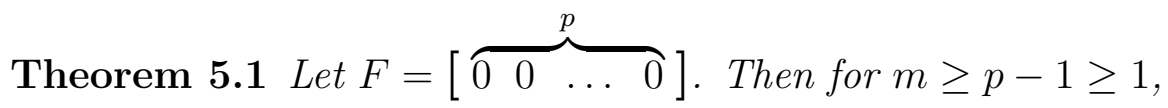

$$
\text { forb }(m, F)=1+\left\lfloor\frac{p m}{2}\right\rfloor \text {. }
$$

Proof: The proof uses a counting argument. We use, successively, the columns with the smallest numbers of 0's. The $m$ row matrix containing the column of 1's together with the $m$ columns containing a single 0 has a single 0 in each row. Adding $k$ columns containg two 0 's each adds an average of $\frac{2 k}{m} 0$ 's in each row. We must have

$$
1+\frac{2 k}{m} \leq p-1,
$$

or,

$$
k \leq \frac{(p-2) m}{2}
$$


for the matrix not to contain $F$. The number of columns is

$$
1+m+k \leq 1+\left\lfloor\frac{p m}{2}\right\rfloor .
$$

With $m \geq p-1$ there are a sufficient number of distinct columns with only two 0's to give a simple $m \times\left(1+\left\lfloor\frac{p m}{2}\right\rfloor\right)$ with no configuration $\mathrm{F}$.

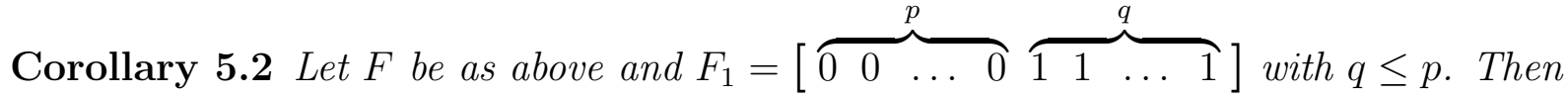
for $m \geq p-1 \geq 1$,

$$
\operatorname{forb}\left(m, F_{1}\right)=\operatorname{forb}(m, F)=1+\left\lfloor\frac{p m}{2}\right\rfloor .
$$

Proof: Let $A$ be a simple $m \times n$ matrix with no configuration $F_{1}$. Each row of $A$ has either fewer than $p$ 0's or fewer than $q 1$ 's. If we take the $(0,1)$-complements of the rows which have fewer than $q 1$ 's, we obtain a simple matrix which has fewer than $p 0$ 's in every row. Thus

$$
\operatorname{forb}\left(m, F_{1}\right) \leq \operatorname{forb}(m, F) .
$$

The reverse inequality is clear.

We use a counting argument as well to establish the following theorem.

Theorem 5.3 Let $F=\left[\begin{array}{llll}\overbrace{0}^{0} & \ldots & 0 \\ 1 & 1 & \ldots & 1\end{array}\right]$. Then, for $m \geq\left\lceil\frac{p+1}{2}\right\rceil$ and $p \geq 3$,

$$
2+\left\lfloor\frac{(p+1) m}{2}\right\rfloor \leq \operatorname{forb}(m, F) \leq 2+\left\lfloor\frac{(p+1) m}{2}+\frac{p-3}{2}+\frac{p-3}{m-2}\right\rfloor .
$$

Proof: We construct a matrix of $m$ rows starting first with the 0's column, the 1's column, the identity and the reverse of the identity. With these we have the configuration $\left[\begin{array}{llll}0 & 0 & 1 & 1 \\ 1 & 1 & 0 & 0\end{array}\right]$ in each pair of rows, with the other entries either $\left[\begin{array}{l}0 \\ 0\end{array}\right]$ 's or $\left[\begin{array}{l}1 \\ 1\end{array}\right]$ 's. We can add the configuration $F^{\prime}=$

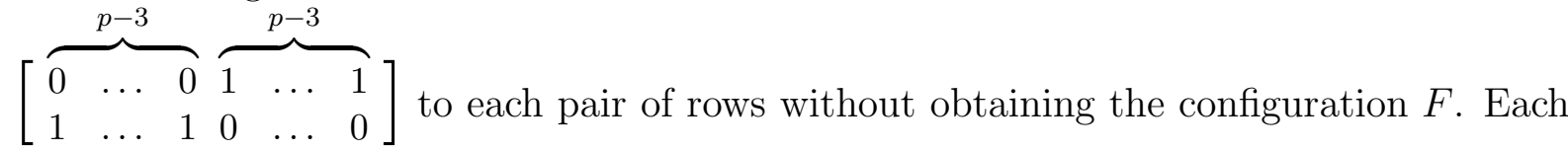
of the columns containing either exactly two 0's or exactly two 1's will contribute $2(m-2)$ $\left[\begin{array}{l}0 \\ 1\end{array}\right]$ configurations to the $\left(\begin{array}{c}m \\ 2\end{array}\right)$ pairs of rows, with columns containing more that two 1's and more than two 0's contributing more. If we add $k$ additional distinct columns without obtaining $F$, we must therefore have

$$
2(m-2) k \leq 2(p-3)\left(\begin{array}{c}
m \\
2
\end{array}\right) .
$$


The number of columns in our matrix is then

$$
2+2 m+k \leq 2+2 m+\left(\frac{p-3}{m-2}\right)\left(\frac{m(m-1)}{2}\right)=2+\frac{(p+1) m}{2}+\frac{p-3}{2}+\frac{p-3}{m-2},
$$

giving the inequality on the right.

To prove the first inequality, we construct a matrix as before, starting with the 0's column, the 1's column, the identity and the reverse of the identity.

Case $1(m \geq p-2)$ : We add $\lfloor(p-3) m / 2\rfloor$ distinct columns with exactly two 0's without adding more than $p-3$ zeros to each row and hence no more than the allowed matrix $F^{\prime}$. Thus

$$
2+2 m+\left\lfloor\frac{(p-3)}{2}\right\rfloor=2+\left\lfloor\frac{(p+1)}{2}\right\rfloor \leq \text { forb }(m, F) .
$$

Case $2((p+1) / 2 \leq m<p-2)$ : We first add all of the $\left(\begin{array}{c}m \\ 2\end{array}\right)$ distinct columns containing two 0's. These add $m-2\left[\begin{array}{ll}0 & 1 \\ 1 & 0\end{array}\right]$ configurations to each pair of rows. We can then add $\lfloor(p-m-1) m / 2\rfloor$ columns with two 1's without adding more than $(p-3-(m-2)) 1$ 's to each row. These $(p-m-1) m / 2+m(m-1) / 2=(p-2) m / 2$ added columns will not have the configuration $F^{\prime}$. Thus, for this case,

$$
2+2 m+\left\lfloor\frac{(p-2)}{2}\right\rfloor=2+\left\lfloor\frac{(p+2)}{2}\right\rfloor \leq \text { forb }(m, F),
$$

which is actually a better lower bound.

Corollary 5.4 For $F$ as above and $m=p-1$ or $m=(p+1) / 2$ with $p$ odd the upper bound is achieved.

Proof: In the first case the columns which contain exactly two 0's have precisely the configuration $F^{\prime}$ with other entries being $\left[\begin{array}{l}0 \\ 0\end{array}\right]$ 's or $\left[\begin{array}{l}1 \\ 1\end{array}\right]$ 's in each pair of rows. In the second case, the columns containing exactly two 0's along with their complements have the same composition for each pair of rows. 